H. Lange $\cdot$ E. Sernesi

\title{
On the Hilbert scheme of a Prym variety
}

\author{
Dedicated to the memory of Fabio Bardelli
}

Received: July 8, 2002

Published online: February 11, 2004 - (c) Springer-Verlag 2004

\section{Introduction}

We work over the field of complex numbers. In this paper we consider the Prym map $\mathcal{P}: \mathcal{R}_{g} \rightarrow \mathcal{A}_{g-1}$ from the moduli space of unramified double covers of projective irreducible and non-singular curves of genus $g \geq 6$ to the moduli space of principally polarized Abelian varieties of dimension $g-1$. If $\pi: \tilde{C} \rightarrow C$ is such a double cover with $C$ non-hyperelliptic, we consider the natural embedding $\tilde{C} \subset P$ (defined up to translation) of $\tilde{C}$ into the Prym variety $P$ of $\pi$ and we study the local structure of the Hilbert scheme Hilb $^{P}$ of $P$ at the point $[\tilde{C}]$ (here and through the paper we adopt the notation [-] for the point of a moduli space or of a Hilbert scheme which parametrizes the object -). We show that this structure is related to the local geometry of the Prym map, or more precisely with the validity of the infinitesimal version of Torelli's theorem for Pryms at $[\pi]$ (see Section 3 for the definitions).

The results we prove are the following.

Proposition. If the infinitesimal Torelli theorem for Pryms holds at $[\pi]$ then $\mathrm{Hilb}^{P}$ is non-singular of dimension $g-1$ at $[\tilde{C}]$ (i.e. $\tilde{C}$ is unobstructed) and the only deformations of $\tilde{C}$ in $P$ are translations.

It is known that if the Clifford index of $C$ is at least 3 then the condition of the proposition is satisfied. Therefore we have, in particular:

Corollary. If $\operatorname{Cliff}(C) \geq 3$ then $\mathrm{Hilb}^{P}$ is non-singular of dimension $g-1$ at $[\tilde{C}]$ (i.e. $\tilde{C}$ is unobstructed) and the only deformations of $\tilde{C}$ in $P$ are translations.

On the other side we have the following result:

Theorem. Assume that the following conditions are satisfied:

(a) the infinitesimal Torelli theorem for Pryms fails at $[\pi]$;

(b) $[\pi]$ is an isolated point of the fibre $\mathcal{P}^{-1}(\mathcal{P}([\pi]))$.

H. Lange: Mathematisches Institut, Bismarckstr. 1 $\frac{1}{2}$, D-91054 Erlangen, Germany, e-mail: lange@mi .uni-erlangen. de

E. Sernesi: Dipartimento di Matematica, Università Roma Tre, L.go S.L. Murialdo 1, 00146 Roma, Italy, e-mail: sernesi@mat .uniroma3 . it 
Then $\tilde{C}$ is obstructed. Moreover the only local deformations of $\tilde{C}$ in $P$ are translations and the only irreducible component of $\mathrm{Hilb}^{P}$ containing $[\tilde{C}]$ is everywhere non-reduced.

Conversely, if $\tilde{C}$ is obstructed then the infinitesimal Torelli theorem for Pryms fails at $[\pi]$.

Using these results we give some examples in which $\tilde{C} \subset P$ is obstructed and some in which we have unobstructedness but the infinitesimal Torelli theorem for Pryms fails. The examples we construct are obtained from double covers belonging to $\mathcal{R}_{6}$ and to $\mathcal{R}_{7}$. For their construction we make use of a result proved in Section 4 which is a slight extension of a theorem of Recillas (see [12]).

The paper is divided into 5 sections. In Section 1 we discuss the Hilbert scheme of curves Abel-Jacobi embedded in their Jacobian. We prove that such curves are obstructed precisely when they are hyperelliptic of genus $g \geq 3$. This case is not relevant for what follows but it is worth keeping in mind the analogies between the two cases. In Section 2 we consider our problem and we study the conditions for the unobstructedness of $[\tilde{C}]$. We use the well-known cohomological description of certain tangent spaces and of maps between them. In Section 3 we relate these results with the infinitesimal Torelli theorem for Pryms and we prove our main result. In Section 4 we give a proof of the extension of Recillas' theorem. The final Section 5 contains the examples.

\section{The case of curves in their Jacobians}

Consider a projective non-singular irreducible curve $C$ of genus $g \geq 2$, let $J C:=$ $\operatorname{PiC}^{0}(C)$ be the Jacobian variety of $C$, and let $j: C \rightarrow J C$ be an Abel-Jacobi map. We want to study the local structure of the Hilbert scheme Hilb ${ }^{J C}$ of $J C$ at $[j(C)]$ (the point parametrizing $j(C)$ ). Since $j$ is an embedding we will identify $C$ with $j(C)$. We have an exact sequence of locally free sheaves on $C$ :

$$
0 \rightarrow T_{C} \rightarrow T_{J C \mid C} \rightarrow N_{C} \rightarrow 0 .
$$

We have a canonical isomorphism $T_{J C \mid C} \cong H^{1}\left(\mathcal{O}_{C}\right) \otimes \mathcal{O}_{C}$ and therefore the cohomology sequence of (1) is:

$$
0 \rightarrow H^{1}\left(\mathcal{O}_{C}\right) \rightarrow H^{0}\left(N_{C}\right) \stackrel{\delta}{\longrightarrow} H^{1}\left(T_{C}\right) \stackrel{\sigma}{\longrightarrow} H^{1}\left(\mathcal{O}_{C}\right) \otimes H^{1}\left(\mathcal{O}_{C}\right) \rightarrow H^{1}\left(N_{C}\right) .
$$

The family of translations of $C$ in $J C$ is parametrized by $J C$ itself, and the map $H^{1}\left(\mathcal{O}_{C}\right) \rightarrow H^{0}\left(N_{C}\right)$ in (2) is precisely the characteristic map of this family at the point 0 . Therefore we have the following Lemma, whose proof is obvious:

Lemma 1.1. The following conditions are equivalent:

(a) Hilb ${ }^{J C}$ is non-singular of dimension $g$ at $[C]$;

(b) $\delta=0$;

(c) $\sigma$ is injective. 
If these conditions are satisfied then the only local deformations of C in JC are translations.

Using this lemma we can prove the following:

Theorem 1.2. Suppose that $C$ has genus $g \geq 3$ :

(a) if $C$ is non-hyperelliptic then Hilb ${ }^{J C}$ is non-singular of dimension $g$ at $[C]$;

(b) if $C$ is hyperelliptic then the connected component of $\mathrm{Hilb}^{J C}$ containing $[C]$ is irreducible of dimension $g$ and everywhere non-reduced with Zariski tangent space of dimension $2 g-2$.

In both cases the only deformations of $C$ in JC are translations.

Proof. The transpose of $\sigma$ is the multiplication map:

$$
\sigma^{\vee}: H^{0}\left(\omega_{C}\right) \otimes H^{0}\left(\omega_{C}\right) \rightarrow H^{0}\left(\omega_{C}^{\otimes 2}\right)
$$

(see [5, Lemma 3]). This map, by Noether's theorem, is surjective if $C$ is nonhyperelliptic and has co-rank $g-2$ if $C$ is hyperelliptic (see [1]). Therefore, in view of Lemma 1.1, part (a) follows.

Now assume that $C$ is hyperelliptic and that $\bar{C} \subset J C$ is a closed subscheme such that $[\bar{C}]$ belongs to the connected component of $\mathrm{Hilb}^{J C}$ containing $[C]$. By the criterion of Matsusaka-Ran (see [7]) $\bar{C}=C_{1} \cup \cdots \cup C_{r}$ is a reduced curve of compact type, and $J C$ and $J C_{1} \times \cdots \times J C_{r}$ are isomorphic as ppav's. Then it follows that $r=1$ and $\bar{C}$ is irreducible and non-singular because $C$ is. Now we apply Torelli's theorem to conclude that $\bar{C}$ is a translate of $C$. It follows that the connected component of $\mathrm{Hilb}^{J C}$ containing $[C]$ is irreducible of dimension $g$ and parametrizes the translates of $C$. On the other hand by (2) we have $h^{0}\left(N_{C}\right)=2 g-2>g$. The conclusion follows.

Theorem 1.2 can be interpreted in terms of the Torelli morphism,

$$
\tau: M_{g} \rightarrow A_{g}
$$

from the moduli stack of projective non-singular curves of genus $g$ to the moduli stack of principally polarized Abelian varieties of dimension $g$. The surjectivity of $\sigma^{\vee}$ is equivalent to that of the multiplication map

$$
S^{2} H^{0}\left(\omega_{C}\right) \rightarrow H^{0}\left(\omega_{C}^{\otimes 2}\right)
$$

which is the codifferential of $\tau$ at $[C]$. Hence the surjectivity of this map is equivalent to the infinitesimal Torelli theorem for $C$ (see [11]). Therefore Theorem 1.2 implies the following:

Corollary 1.3. $C$ is unobstructed in JC if and only if the infinitesimal Torelli theorem holds for $C$.

Remarks. (i) The proof of Theorem 1.2(a) has already appeared in [5], but the argument does not appear to be complete. A proof is also given in [3] using the semiregularity map, but it is more complicated; moreover, the semiregularity map does not seem to be able to detect what happens in case (b).

(ii) In the case $g=2$ we have that $C$ is unobstructed in $J C$ because the semiregularity map $H^{1}\left(N_{C}\right) \rightarrow H^{2}\left(\mathcal{O}_{J C}\right)$ is injective since $H^{1}\left(\mathcal{O}_{J C}(C)\right)=0$ by the ampleness of $C$ in $J C$. 


\section{The Hilbert scheme of the Prym variety at $[\tilde{\mathbf{C}}]$}

Let now $\pi: \tilde{C} \rightarrow C$ be an unramified double cover of a projective non-singular irreducible curve $C$ of genus $g \geq 3$, so that $\tilde{C}$ has genus $\tilde{g}=2 g-1$. Let $\eta \in P i c^{0}(C)$ be the 2-division point corresponding to $\pi$. We have a canonical isogeny $J \tilde{C} \rightarrow J C \times P$, where $P$ is the Prym variety of $\pi$. Throughout this section we assume $C$ to be non-hyperelliptic. Under this hypothesis we have an embedding $\alpha: \tilde{C} \rightarrow P$ which is obtained as the composition

$$
\tilde{C} \rightarrow J \tilde{C} \rightarrow J C \times P \rightarrow P
$$

(see [7]). We will identify $\tilde{C}$ with $\alpha(\tilde{C})$. We want to study the Hilbert scheme Hilb $^{P}$ locally at the point $[\tilde{C}]$.

In analogy with the situation studied in Section 1, we consider the exact sequence of locally free sheaves on $\tilde{C}$,

$$
0 \rightarrow T_{\tilde{C}} \rightarrow T_{P \mid \tilde{C}} \rightarrow N_{\tilde{C}} \rightarrow 0 .
$$

We have a canonical isomorphism $T_{P \mid \tilde{C}} \cong H^{1}(C, \eta) \otimes \mathcal{O}_{\tilde{C}}$ so that the cohomology sequence of (3) becomes

$$
\begin{aligned}
& 0 \longrightarrow H^{1}(C, \eta) \longrightarrow H^{0}\left(\tilde{C}, N_{\tilde{C}}\right) \stackrel{\delta}{\longrightarrow} H^{1}\left(\tilde{C}, T_{\tilde{C}}\right) \\
& \stackrel{\sigma}{\longrightarrow} H^{1}(C, \eta) \otimes H^{1}\left(\tilde{C}, \mathcal{O}_{\tilde{C}}\right) \longrightarrow H^{1}\left(\tilde{C}, N_{\tilde{C}}\right) .
\end{aligned}
$$

Along the same lines of Section 1 we can state the following:

Lemma 2.1. The following conditions are equivalent:

(a) Hilb ${ }^{P}$ is non-singular of dimension $g-1$ at $[\tilde{C}]$;

(b) $\delta=0$;

(c) $\sigma$ is injective.

If these conditions are satisfied then the only local deformations of $\tilde{C}$ in $P$ are translations.

In order to understand the conditions of Lemma 2.1 we must study the map $\sigma$, or equivalently, its transpose $\sigma^{\vee}$. We have canonical isomorphisms:

$$
H^{1}\left(\tilde{C}, \mathcal{O}_{\tilde{C}}\right)^{\vee} \cong H^{0}\left(\tilde{C}, \omega_{\tilde{C}}\right) \cong H^{0}\left(C, \omega_{C}\right) \oplus H^{0}\left(C, \omega_{C} \otimes \eta\right)
$$

and

$$
H^{1}\left(\tilde{C}, T_{\tilde{C}}\right)^{\vee} \cong H^{0}\left(\tilde{C}, \omega_{\tilde{C}}^{\otimes 2}\right) \cong H^{0}\left(C, \omega_{C}^{\otimes 2} \otimes \eta\right) \oplus H^{0}\left(C, \omega_{C}^{\otimes 2}\right)
$$

corresponding to the decompositions into +1 and -1 eigenvalues under the action induced by the involution on $\tilde{C}$. Hence

$$
\begin{gathered}
\sigma^{\vee}: H^{0}\left(C, \omega_{C} \otimes \eta\right) \bigotimes\left[H^{0}\left(C, \omega_{C}\right) \oplus H^{0}\left(C, \omega_{C} \otimes \eta\right)\right] \\
\rightarrow H^{0}\left(C, \omega_{C}^{\otimes 2} \otimes \eta\right) \oplus H^{0}\left(C, \omega_{C}^{\otimes 2}\right),
\end{gathered}
$$


and it is induced by multiplication of sections [2, p. 382]. Therefore, after decomposing the domain of $\sigma^{\vee}$ as

$$
\begin{gathered}
H^{0}\left(C, \omega_{C} \otimes \eta\right) \bigotimes\left[H^{0}\left(C, \omega_{C}\right) \oplus H^{0}\left(C, \omega_{C} \otimes \eta\right)\right] \\
=\left[H^{0}\left(C, \omega_{C} \otimes \eta\right) \otimes H^{0}\left(C, \omega_{C}\right)\right] \bigoplus\left[H^{0}\left(C, \omega_{C} \otimes \eta\right) \otimes H^{0}\left(C, \omega_{C} \otimes \eta\right)\right],
\end{gathered}
$$

we see that $\sigma^{\vee}=\mu \oplus v$, where

$$
\mu: H^{0}\left(C, \omega_{C} \otimes \eta\right) \otimes H^{0}\left(C, \omega_{C}\right) \rightarrow H^{0}\left(C, \omega_{C}^{\otimes 2} \otimes \eta\right)
$$

and

$$
v: H^{0}\left(C, \omega_{C} \otimes \eta\right) \otimes H^{0}\left(C, \omega_{C} \otimes \eta\right) \rightarrow H^{0}\left(C, \omega_{C}^{\otimes 2}\right) .
$$

The following lemma is well known (see [2, Prop. 7.7]):

Lemma 2.2. The sheaf $\omega_{C} \otimes \eta$ is not very ample if and only if there exist points $x, y, z, t \in C$ such that $\eta \cong \mathcal{O}_{C}(x+y-z-t)$. If these conditions are satisfied then the map $v$ is not surjective.

Proposition 2.3. (i) In each of the following cases the map $\mu$ is surjective:

(a) $C$ is not bi-elliptic;

(b) $v$ is surjective;

(ii) if C is not bi-elliptic then $\operatorname{cork}\left(\sigma^{\vee}\right)=\operatorname{cork}(v)$. In particular if $C$ is not bi-elliptic the surjectivity of $\sigma^{\vee}$ is equivalent to the surjectivity of $v$.

Proof. (i) Note first that in both cases (a) and (b) the linear series $\left|\omega_{C} \otimes \eta\right|$ is base point free and is not composed with an involution: in fact, in case (a) since $C$ is not hyperelliptic, $\left|\omega_{C} \otimes \eta\right|$ is base point free; moreover if it were composed with an involution then, since $\operatorname{deg}\left(\omega_{C} \otimes \eta\right)=2 g-2$, the morphism

$$
\phi_{\eta}: C \rightarrow \mathbf{P}^{g-2}
$$

would be of degree 2 onto a curve of degree $g-1$, which has genus $\leq 1$, a contradiction. In the case (b) the assertion is true by Lemma 2.2.

Let $\underline{b}:=P_{1}+\cdots+P_{g-3} \in C^{(g-3)}$ be general. Consider the exact sequence on $C$

$$
0 \rightarrow \omega_{C} \otimes \eta(-\underline{b}) \rightarrow \omega_{C} \otimes \eta \rightarrow \mathcal{T} \rightarrow 0,
$$

where $\mathcal{T}$ is a torsion sheaf supported on $\underline{b}$. Multiplying firstly by $H^{0}\left(\omega_{C}\right)$ and taking cohomology, and secondly by $\omega_{C}$ and taking cohomology, we obtain the following commutative diagram with exact rows, where the vertical maps are given by multiplication:

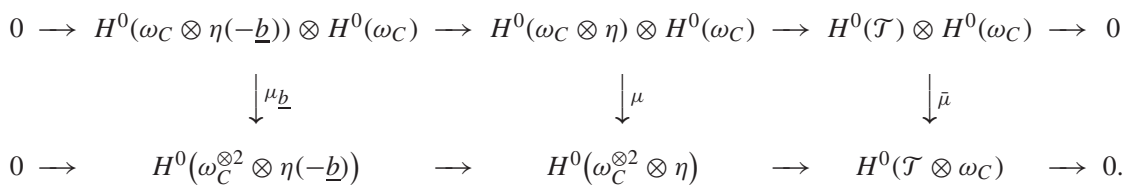


Since $\left|\omega_{C} \otimes \eta\right|$ is not composed with an involution and $\underline{b}$ is generic, $\omega_{C} \otimes \eta(-\underline{b})$ is base point free, and by the base point free pencil trick we find

$$
\operatorname{ker}\left(\mu_{\underline{b}}\right)=H^{0}(\underline{b} \otimes \eta)=0,
$$

hence

$$
\operatorname{rk}\left(\mu_{\underline{b}}\right)=2 g=h^{0}\left(\omega_{C}^{\otimes 2} \otimes \eta(-\underline{b})\right),
$$

i.e. $\mu_{\underline{b}}$ is surjective. On the other hand $\bar{\mu}$ is surjective because $\omega_{C}$ is globally generated. The conclusion follows from the above diagram.

(ii) follows immediately from part (i) and from the relation between the maps $\sigma, \mu, \nu$.

Collecting all we have said so far we can state the following:

Corollary 2.4. If $v$ is surjective then $\mathrm{Hilb}^{P}$ is non-singular of dimension $g-1$ at $[\tilde{C}]$ (i.e. $\tilde{C}$ is unobstructed) and the only local deformations of $\tilde{C}$ in $P$ are translations.

As an application we can prove the following:

Corollary 2.5. If $\operatorname{Cliff}(C) \geq 3$ then $\mathrm{Hilb}^{P}$ is non-singular of dimension $g-1$ at $[\tilde{C}]$ (i.e. $\tilde{C}$ is unobstructed) and the only local deformations of $\tilde{C}$ in $P$ are translations.

Proof. It follows easily from a result of [6] (see e.g. [8]) that if $\operatorname{Cliff}(C) \geq 3$ then the map $v$ is surjective. Therefore the corollary follows from Corollary 2.4 .

\section{Hilb ${ }^{P}$ and the infinitesimal Torelli theorem for Pryms}

We keep the notations of Section 2. Consider the Prym morphism

$$
\mathcal{P}: \mathcal{R}_{g} \rightarrow \mathcal{A}_{g-1},
$$

which goes from the coarse moduli scheme of étale double covers of curves of genus $g$ to the coarse moduli scheme of ppav of dimension $g-1, g \geq 6$. These schemes have singularities due to the presence of automorphisms of the objects they classify. Therefore if we want to study the infinitesimal properties of $\mathcal{P}$ it is more natural to consider the corresponding moduli stacks $R_{g}, A_{g-1}$. The Prym construction defines a morphism of stacks

$$
\operatorname{Pr}: R_{g} \rightarrow A_{g-1} \text {. }
$$

Then the map $v$ considered in Section 2 coincides with the co-differential of $\operatorname{Pr}$ at $[\pi]$ (see [2, Proposition 7.5], which implies this statement modulo obvious modifications). Therefore the surjectivity of $v$ is equivalent to Pr being an immersion at $[\pi]$ (see $[2,7.6])$. In this case we say that the infinitesimal Torelli theorem for Pryms holds at $[\pi]$, according to the terminology most commonly used nowadays. In view of Corollary 2.4 we can therefore state the following: 
Proposition 3.1. If the infinitesimal Torelli theorem for Pryms holds at $[\pi]$ then Hilb $^{P}$ is non-singular of dimension $g-1$ at $[\tilde{C}]$ (i.e. $\tilde{C}$ is unobstructed) and the only local deformations of $\tilde{C}$ in $P$ are translations.

In the case $\operatorname{Cliff}(C) \leq 2$ the infinitesimal Torelli theorem for Pryms in general fails, i.e. in general $v$ is not surjective. Our next goal is to relate the obstructedness of $\tilde{C}$ in $P$ to the failure of the infinitesimal Torelli theorem for Pryms. The main result of this section is the following:

Theorem 3.2. Assume that the following conditions are satisfied:

(a) the infinitesimal Torelli theorem for Pryms fails at $[\pi]$;

(b) $[\pi]$ is an isolated point of the fibre $\mathcal{P}^{-1}(\mathcal{P}([\pi]))$.

Then $\tilde{C}$ is obstructed. Moreover the only local deformations of $\tilde{C}$ in $P$ are translations; in particular the only irreducible component of $\mathrm{Hilb}^{P}$ containing $[\tilde{C}]$ is everywhere non-reduced of dimension $g-1$.

Conversely, if $\tilde{C}$ is obstructed then the infinitesimal Torelli theorem fails at $[\pi]$.

Proof. By (a) the map $\delta$ in the exact sequence (4) is non-zero. Assume by contradiction that $[\tilde{C}]$ is unobstructed. Then we can find a non-singular curve $S \subset H_{i l b}{ }^{P}$ passing through $[\tilde{C}]$ such that $\delta\left(T_{S,[\tilde{C}]}\right) \neq 0$. This condition implies that the functorial morphism $S \rightarrow \mathcal{M}_{\tilde{g}}$ defined by the family of curves $\mathcal{C} \rightarrow S$ (which can be assumed to be smooth) is not constant and therefore this family does not consist of curves all isomorphic to $\tilde{C}$. But this is impossible: in fact for each curve $\tilde{C}^{\prime}$ in the family we have

$$
\tilde{C}^{\prime} \equiv_{\text {num }} \tilde{C} \equiv_{\text {num }} \frac{2}{(g-2) !} \Xi^{g-2},
$$

so that by a theorem of Welters (see [13]) there is an étale double cover $\pi^{\prime}: \tilde{C}^{\prime} \rightarrow C^{\prime}$, $(P, \Xi)$ is the Prym variety of $\pi^{\prime}$ and $\tilde{C}^{\prime}$ is Prym embedded. But this contradicts condition (b) if $\tilde{C}^{\prime}$ is not isomorphic to $\tilde{C}$ because $\left[\pi^{\prime}\right] \in \mathcal{P}^{-1}(\mathcal{P}([\pi]))$.

This analysis also shows that locally the only deformations of $\tilde{C}$ in $P$ are translations; and since $\delta \neq 0$ the Zariski tangent space of $\mathrm{Hilb}^{P}$ at $[\tilde{C}]$ has dimension larger than $g-1$. This also proves the last assertion.

The converse is a special case of Proposition 3.1 .

The theorem does not say anything in the case when $[\pi]$ is a non-isolated point of the fibre $\mathcal{P}^{-1}(\mathcal{P}([\pi]))$. We will see in Section 5 that in this case there are examples where $[\tilde{C}]$ is unobstructed.

\section{Further considerations}

A theorem of Recillas [12] says that if $\pi: \tilde{C} \rightarrow C$ is a double cover with $C$ trigonal (but not hyperelliptic) of genus $g$ then $\mathcal{P}([\pi])=[J X]$ with $X$ a 4-gonal curve, and the pair $\left(X, g_{4}^{1}\right)$ is uniquely determined. A consequence of this result 
and of a theorem of Mumford [9], which gives a list of the Prym varieties which are Jacobians, is that

$$
\mathcal{P}^{-1}([J X])=W_{4}^{1}(X)
$$

set-theoretically if $g-1 \geq 6$. This says in particular that for $g \geq 11$ the Prym map is $1-1$ on $\mathcal{R}_{g, T}$ (= the locus of étale double covers of trigonal curves): this follows from the fact that $W_{4}^{1}(X)$ consists of at most one point if $g(X) \geq 10$. If $g-1=5$ then (5) is not true but we have a strict inclusion $\supset$ (see Section 4). The following proposition gives some further information which will suffice for some applications.

Proposition 4.1. Assume that $X$ is a non-singularirreducible curve of genus $g-1$ $\geq 5$, non-hyperelliptic nor trigonal, and such that every $g_{4}^{1}$ on $X$ has no divisors of the form $2 P+2 Q$ or $4 P$ and let $\pi: \tilde{C} \rightarrow C$ be an unramified double cover, with $C$ trigonal of genus $g$, such that $\mathcal{P}([\pi])=[J X]$. Then there is a canonical isomorphism between the kernel of the differential of $\operatorname{Pr}$ at $[\pi]$ and the Zariski tangent space of $W_{4}^{1}(X)$ at the line bundle L corresponding to $\pi$.

Proof. Since $X$ is not trigonal we may view $W_{4}^{1}(X)$ as parametrizing 4-1 morphisms of $X$ into $\mathbf{P}^{1}$. Let $\varphi: X \rightarrow \mathbf{P}^{1}$ be the $4-1$ cover defined by $L$.

Let $B=\operatorname{Spec}(\mathbf{C}[\epsilon])$ and consider a family of deformations of $\varphi$ parametrized by $B$ :

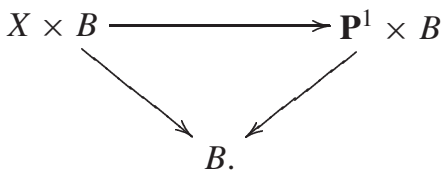

To this family we can associate a family of deformations of $\pi$ just extending Recillas's construction, as follows. Consider the second relative symmetric product over $\mathbf{P}^{1} \times B$ :

$$
\tilde{\mathrm{C}}:=S_{\mathbf{P}^{1} \times B}^{(2)}(X \times B),
$$

which comes endowed with an induced family of morphisms of degree 6

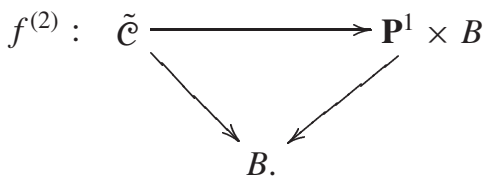

On $\tilde{\mathcal{C}}$ there is a natural involution $\iota$ commuting with the projection to $B$. Letting $\mathcal{C}=\tilde{\mathcal{C}} / \iota$, we obtain a family parametrized by $B$ :

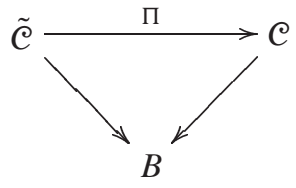


such that $f^{(2)}$ factors through $\Pi$. Therefore (6) is a first-order deformation of $\pi$; moreover (6) is contained in $\mathcal{P}^{-1}([J X])$ by construction and therefore it is an element of $\operatorname{ker}\left(d \operatorname{Pr}_{[\pi]}\right)$.

Conversely, assume a family (6) given, and assume that (6) is contained in $\operatorname{ker}\left(d \operatorname{Pr}_{[\pi]}\right)$. Then we also have a family of triple covers of $\mathbf{P}^{1}$ :

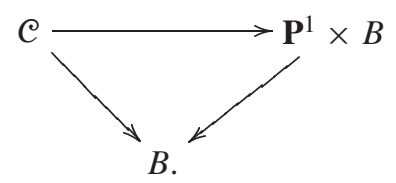

Correspondingly we have an inclusion $\mathbf{P}^{1} \times B \subset S_{B}^{(3)}(\mathcal{C})$, and an étale morphism of degree 8

$$
\Pi^{(3)}: S_{B}^{(3)}(\tilde{\mathcal{C}}) \rightarrow S_{B}^{(3)}(\mathcal{C})
$$

induced by $\Pi$. Let $\mathscr{D}:=\Pi^{(3)-1}\left(\mathbf{P}^{1} \times B\right)$. The involution $\iota$ on $\tilde{\mathcal{C}}$ induces an involution on $S_{B}^{(3)}(\tilde{\mathcal{C}})$ which commutes with $\Pi^{(3)}$ and induces an involution on $\mathcal{D}$. We obtain a commutative diagram:

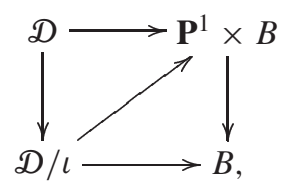

where the diagonal morphism defines a family of deformations of $X$ with an assigned $g_{4}^{1}$ on the family. The assumption that (6) is contained in $\operatorname{ker}\left(d \operatorname{Pr}_{[\pi]}\right)$ means that the family of ppav obtained as Pryms of the family (6) is the family of Jacobians of $\mathscr{D} / \iota \rightarrow B$ and that it is trivial. By the infinitesimal Torelli theorem for Jacobians $\mathscr{D} / \iota \rightarrow B$ is the trivial family as well. Therefore diagram (7) gives us a family of deformations of $\varphi$.

\section{Examples}

5.1. Let $\bar{X} \subset \mathbf{P}^{2}$ be an irreducible sextic having 4 distinct nodes $N_{1}, \ldots, N_{4}$, and let $X$ be the normalization of $\bar{X}$, which has genus 6 . If no three among $N_{1}, \ldots, N_{4}$ are on a line then $W_{4}^{1}(X)$ consists of five non-singular points, the $g_{4}^{1}$ 's cut by the four pencils of lines through each of the nodes and by the pencil of conics containing $N_{1}, \ldots, N_{4}$. From Proposition 4.1 it then follows that the infinitesimal Prym-Torelli theorem holds at the five double covers $\pi: \tilde{C} \rightarrow C$ of trigonal curves of genus 7 such that $\mathcal{P}([\pi])=[J X]$.

Assume now that $\bar{X}$ has three of the nodes, say $N_{1}, N_{2}, N_{3}$, on a line. Then the $g_{4}^{1}$ defined by $N_{4}$ and that defined by the pencil of conics are identified to a unique element $L$ of $W_{4}^{1}(X)$ with a 1-dimensional Zariski tangent space. Applying Proposition 4.1 to this case we see that the infinitesimal Prym-Torelli theorem fails at the double cover $\pi$ corresponding to $(X, L)$ in the fibre $\mathcal{P}^{-1}([J X])$. Moreover, 
since equality (5) implies that $\mathcal{P}^{-1}([J X])$ is finite, from Theorem 3.2 we deduce that $\tilde{C}$ is obstructed in $J X$ and that the only component of $H_{i l b}{ }^{P}$ containing $[\tilde{C}]$ is everywhere non-reduced and consists of translates of $[\tilde{C}]$.

A count of parameters shows that in this way we get a 14-dimensional locus where the infinitesimal Prym-Torelli theorem fails inside the 18-dimensional space $\mathcal{R}_{7}$.

5.2. Let's consider the Prym map $\mathcal{P}: \mathcal{R}_{6} \rightarrow \mathcal{A}_{5}$. This case has been extensively studied in [4] and offers a wide variety of examples, but it is not yet completely understood from the point of view of the infinitesimal Prym-Torelli theorem. Recall that both domain and co-domain are irreducible of dimension 15. Some loci where the infinitesimal Prym-Torelli theorem fails are the following.

5.2.1. Consider a non-singular curve $C \subset \mathbf{P}^{4}$ obtained as a general hyperplane section of a Reye congruence in $\mathbf{P}^{5}$, i.e. of an Enriques surface $S$ of degree 10 contained in a non-singular quadric. Then $C$ is a curve of genus 6 , embedded with a Prym canonical linear series $|\omega \otimes \eta|$; since $C$ is contained in a quadric it follows that the map $v$ is not surjective, and therefore the infinitesimal Prym-Torelli theorem fails at the double cover $\pi: \tilde{C} \rightarrow C$ associated to $\eta$.

Naranjo-Verra proved that the fibre $\mathcal{P}^{-1}(\mathcal{P}([\pi]))$ is discrete [10]. Therefore from Theorem 3.2 it follows that $H_{i l b}{ }^{P}$ is obstructed at $\tilde{C}$.

Note that a count of parameters shows that the locus of double covers $\pi$ constructed in this way has dimension $14=9+5$ ( 9 for the moduli of Enriques surfaces and 5 for the hyperplane sections), i.e. it is a divisor in $\mathcal{R}_{6}$. In particular it follows that a general such curve $C$ is not trigonal since trigonal curves depend on 13 parameters.

5.2.2. Consider an irreducible sextic $\bar{C} \subset \mathbf{P}^{2}$ with four nodes such that two of its bi-tangents meet in one of the nodes, say $N$. Then the normalization $C$ has genus 6 and the $g_{4}^{1}$ defined by the pencil of lines through $N$ has two divisors of the form $2 P+2 Q$. It follows that there is a 2-division point $\eta \in \operatorname{Pic}(C)$ such that $\omega \otimes \eta$ is not very ample and the map $v$ is not surjective (use Lemma 2.2). Therefore the infinitesimal Prym-Torelli theorem fails at the double cover $\pi: \tilde{C} \rightarrow C$ associated to $\eta$. The locus in $\mathcal{R}_{6}$ defined by this family of examples is disjoint from the previous one because there the line bundles $\omega \otimes \eta$ were very ample. It is not clear to us what the dimensions of the fibres $\mathcal{P}^{-1}(\mathcal{P}([\pi]))$ are and therefore whether $[\tilde{C}]$ is obstructed in this case.

5.2.3. Another locus where the infinitesimal Prym-Torelli fails is $\mathcal{R}_{6, T}$, the locus of double covers of trigonal curves. It has dimension 13, and the restriction of $\mathcal{P}$ to $\mathcal{R}_{6, T}$ has general fibre of dimension 1 , as it follows from Recillas's theorem recalling that $W_{4}^{1}(X)$ for a curve $X$ of genus 5 has dimension 1 .

What is interesting here is that $W_{4}^{1}(X)=\Theta_{\text {sing }}$, the singular locus of the theta divisor of $J X$ : for a general $X$ this is a non-singular curve of genus 11 which has an involution $\iota$ with quotient a non-singular plane quintic $C$. The double cover $\pi: W_{4}^{1}(X) \rightarrow C$ is associated with a 2 -division point $\eta$ such that $\mathcal{O}(1) \otimes \eta$ is 
an even theta-characteristic (see [4] for details). Moreover $\mathcal{P}([\pi])=[J X]$ again, by [9]. Therefore we see that for a general $X$ of genus 5 we have

$$
\mathcal{P}^{-1}([J X])=W_{4}^{1}(X) \cup\{[\pi]\} .
$$

In particular the fibre of $\mathcal{P}$ is not equi-dimensional. Moreover $\nu$ is surjective at $[\pi]$ (see [4, part II, Section 5]) and therefore the curve $W_{4}^{1}(X)=\Theta_{\text {sing }}$ is unobstructed in $J X$. Note that this gives an example of a double cover $\pi$ of a curve of Clifford index 1 (namely a non-singular plane quintic) at which the infinitesimal PrymTorelli theorem holds.

Note also that, since cork $(v)$ is 1 -dimensional if $\pi: \tilde{C} \rightarrow C$ is a double cover of a general trigonal curve of genus 6 , we have that $\operatorname{cork}(\sigma)$ is 1 -dimensional as well, by Proposition 2.3 (clearly a general trigonal $C$ is not bi-elliptic). Therefore $\delta$ has rank 1 and

$$
h^{0}\left(\tilde{C}, N_{\tilde{C}}\right)=g .
$$

With some extra effort one can easily show that in this case $\tilde{C}$ is unobstructed in $J X$. In fact consider a small (1-dimensional) neighborhood $A$ of $[\pi]$ in the fibre $\mathcal{P}^{-1}([J X])$ and let

$$
\tilde{\mathrm{e}} \subset J X \times A
$$

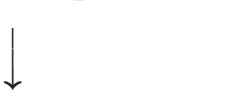

A

be the corresponding 1-parameter family of deformations of $\tilde{C}$ in $J X$. Since this family has varying moduli, in the exact sequence (4) we have $0 \neq \delta(v) \in$ $H^{1}\left(\tilde{C}, T_{\tilde{C}}\right)$ if $v \neq 0$ is a tangent vector to $A$ at the point $a_{0}$ parametrizing $\tilde{C}$, and $\delta(v)$ generates $\operatorname{Im}(\delta)$. Now consider a small neighborhood $B$ of 0 in $J X$ and build a new family:

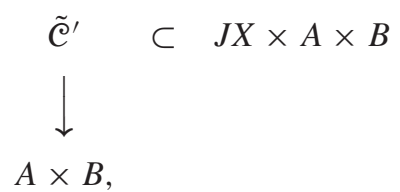

whose fibre over $(a, b)$ is the curve $t_{b}^{*}\left(\tilde{\mathcal{C}}_{a}\right)$, i.e. the translate by $b$ of the fibre $\tilde{\mathcal{C}}_{a}$ of the family (8). It is clear that the characteristic map

$$
T_{A \times B,\left(a_{0}, 0\right)} \rightarrow H^{0}\left(\tilde{C}, N_{\tilde{C}}\right)
$$

is an isomorphism, proving that $\tilde{C}$ is unobstructed. Note that $h^{0}\left(\tilde{C}, N_{\tilde{C}}\right)>g-1$ in this case, and $\tilde{C}$ has non-trivial moduli.

\section{References}

1. Arbarello, E., Cornalba, M., Griffiths, P.A., Harris, J.: Geometry of Algebraic Curves I. Springer Grundlehren 267 (1985)

2. Beauville, A.: Variétés de Prym et Jacobiennes intermédiaires. Ann. Sci. Éc. Norm. Supér., IV. Sér. 10, 309-391 (1977) 
3. Bloch, S.: Semi-regularity and De Rham cohomology. Invent. Math. 17, 51-66 (1972)

4. Donagi, R., Smith, R.C.: The structure of the Prym map. Acta Math. 146, 25-102 (1982)

5. Griffiths, $\mathrm{Ph}$.: Some remarks and examples on continuous systems and moduli. J. Math. Mech. 16, 789-802 (1967)

6. Green, M., Lazarsfeld, R.: On the projective normality of complete linear series on an algebraic curve. Invent. Math. 83, 73-90 (1986)

7. Lange, H., Birkenhake, Ch.: Complex Abelian Varieties. Springer Grundlehren 302 (1992)

8. Lange, H., Sernesi, E.: Quadrics containing a Prym-canonical curve. J. Algebr. Geom. 5, 387-399 (1996)

9. Mumford, D.: Prym varieties I. In: Contributions to Analysis, New York: Academic Press 1974

10. Naranjo, J.C., Verra, A.: In preparation

11. Oort, F., Steenbrink, J.: On the local Torelli problem for algebraic curves. J. Geom. Alg. Angers 1979, Sijhoff and Noordhoff (1980), 157-204

12. Recillas, S.: Jacobians of curves with a $g_{4}^{1}$ are Prym varieties of trigonal curves. Bol. Soc. Mat. Mex., III. Ser. 19, 9-13 (1974)

13. Welters, G.: Curves of twice the principal class on principally polarized Abelian varieties. Indag. Math., New Ser. 94, 87-109 (1987) 